\title{
A study method for possible breakdown of boiler tank for dangerous goods in an emergency collision
}

\author{
S.V. Bespalko ${ }^{1, *}$, P.S. Grigoriev ${ }^{1}$, M.I. Ruzmatov ${ }^{2}$, and Sh.R. Ibodulloev ${ }^{2}$ \\ ${ }^{1}$ Russian University of Transport, 127994, Moscow, Russia \\ ${ }^{2}$ National University of Uzbekistan, 100174, Tashkent, Uzbekistan
}

\begin{abstract}
An impact to the boiler of a railway tank car in an emergency in the event of a car derailment and falling onto a track has been considered. In this case, boiler breakdown, spillage of the transported cargo and fire can occur. When developing a method, the following objectives have been solved: - development of a method for determining a stress-deformed state (SDS) of a cylindrical shell at the initial elastic stage of deformation; development of a method for calculating the deformation energy for the boiler shell when the yield point in the impact zone is reached; development of a method for estimating the conditions for the beginning of the limiting state (shell destruction). For the shell behavior to be modeled, a moment theory of shells has been used with taking into account the nonlinear terms in stress-strain deformations. The energy principles in the form of the Lagrange principle have been applied to compile a resolving system of equations. The onset of the limiting state is taken according to the condition of a cut along the striker perimeter. A system of the nonlinear equations at the first stage and a transcendental equation at the second one has been solved using numerical methods. The proposed method makes it possible to develop methods of protection in terms of the choice of methods for attaching add-on elements to the boiler.
\end{abstract}

\section{Introduction}

An emergency situation in which a railway tank boiler undergoes an impact is considered. Add-on elements welded to the boiler can act as an impacting body (striker) in the event of a car derailing from the rails and falling onto the railway road. This emergency situation is very dangerous since it can cause boiler breakdown followed by leakage of transported cargo and fire.

A method developed for modeling the boiler behavior under this impact will make it possible to develop reliable protection methods for attaching the add-on elements to the boiler. Thus, the development of a method for estimating the breakdown conditions of the boiler shell under emergency collision is relevant and topical for the design engineering of tanks for dangerous goods.

*Corresponding author: besp-alco@yandex.ru 
It should be noted that this problem has not been fully studied. In particular, in the work [1] the standard collision process that takes place during shunting operations was considered. In the works $[5,6,7]$ the process of an emergency impact of a foreign body into the tank boiler was studied. In this case, the initial elastic phase of shell deformation during dent formation was not taken into account.

There are approaches to studying an emergency impact at the bottom in which the boiler is approximated not on the basis of the shell theory but by a rod system. The paper [8] describes a method for protecting the boiler bottom in case of an emergency impact by a disengaged automatic coupler of a neighboring car. However, this method does not allow to the boiler to be protected from impacts to its cylindrical part, for example, in case of an emergency fall of the tank.

\section{Goal and objectives}

The goal of this work is to create a method for modeling the behavior of the tank boiler shell during an emergency collision.

To achieve this goal, it is necessary to resolve the following objectives:

- development of a method for determining a stress-deformed state (SDS) of the cylindrical shell at the initial elastic stage of deformation;

- development of a method for calculating the deformation energy of the boiler shell when reaching the yield point in the impact zone of the striker;

- development of a method for estimating the conditions for the beginning of the limiting state (shell destruction).

\section{Materials and methods}

The method is based on the following assumptions.

1. The cylindrical region of the shell is considered; outside this region the shell deformations are neglected.

2. The shell material is assumed to be solid and isotropic.

3. Only radial displacements of the shell are taken into account.

4. In the phase of the leakage appearance, a dent in the shell is approximated by a rectangular truncated pyramid.

5. In the phase of plastic deformations, plastic hinges appear along the perimeters of the bases and on the side faces. Membrane plastic deformations occur on the lateral faces of the dent.

6. The boiler thickness is assumed to be constant in the process of deformation.

7. The shell inertia is neglected.

8. The impact direction is assumed to be normal to the shell.

9. The beginning of the limiting state is characterized by cutting out of the shell a rectangle corresponding to the striker sizes.

10. The deformation process is divided into two successive phases:

- elastic phase with a constant value of the elastic modulus and

- plastic one in which deformation occurs only when there is a yield point owing to stresses.

When developing the method, the following methods were applied.

When modeling the stress-deformed state of the shell in the elastic phase of deformation, a moment theory of shells [2] with consideration of the nonlinear terms was used to calculate stress-strain deformation [3]. 
In the elastic phase of deformation, a resolving system of the equations is based on the Lagrange principle [4].

In the phase of plastic deformations, the total deformation energy of the shell is calculated taking into account the action of stresses equal to the yield point on the faces and edges of the dent $[5,6,7]$.

The system of nonlinear equations at the first stage and the transcendental equation at the second one is solved by numerical methods.

\section{Results}

\subsection{Modeling the elastic phase of deformation}

Consider the initial elastic phase of the shell deformation. The unfolded shell is shown in Fig. 1. Here $\mathrm{a}$ and $\mathrm{Rb}$ are the sizes of the shell area under study along the longitudinal $\mathrm{x}$ and circumferential $\sqcup$ coordinates, respectively. The contact force Fk from the striker is evenly distributed over the area with the sizes xk and R $\sqcup \mathrm{k}$.

In accordance with the accepted assumptions, geometric nonlinearity is taken into account in the initial equations. The geometric ratios of the shell are as follows $[2,3]$ :

$$
\left\{\begin{array}{l}
\varepsilon_{1}=\frac{1}{2}\left(\frac{\partial w}{\partial x}\right)^{2} ; \varepsilon_{2}=\frac{w}{R}+\frac{1}{2 R^{2}}\left(\frac{\partial w}{\partial \beta}\right)^{2} ; \omega=\frac{\partial w}{\partial x} \frac{\partial w}{R \partial \beta} \\
\kappa_{1}=-\frac{\partial^{2} w}{\partial x^{2}} ; \kappa_{2}=-\frac{\partial^{2} w}{R^{2} \partial \beta^{2}} ; \tau=-\frac{\partial^{2} w}{R \partial x \partial \beta},
\end{array}\right.
$$

where $\sqcup 1, \sqcup 2$ are the stress-strain deformations in the directions of the generatrix and the circle, respectively; $\sqcup \sqcup$ is the shear deformation; $\sqcup 1, \sqcup 2$ are the deformations of the change in curvature in the planes of the generatrix and the guide, respectively; $\sqcup \sqcup$ is the torsional deformation; $\mathrm{w}$ is the radial displacement; $\mathrm{R}$ is the radius of the median surface; $\mathrm{x}$ is the longitudinal coordinate; $\beta$ is the circumferential (angular) coordinate.

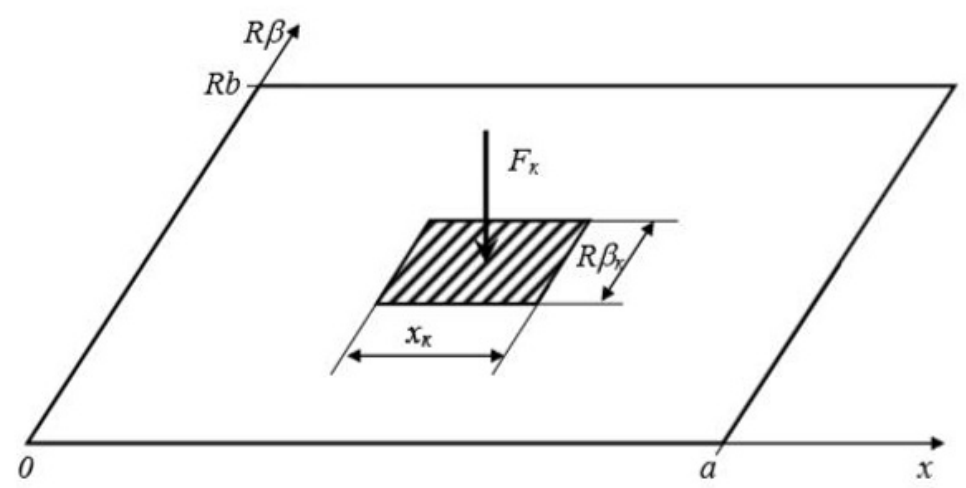

Fig. 1. The shell area under study.

The physical relations of the shell theory [2] have the form:

$$
\left\{\begin{array}{l}
N_{1}=B\left(\varepsilon_{1}+\mu \varepsilon_{2}\right) ; N_{2}=B\left(\varepsilon_{2}+\mu \varepsilon_{1}\right) ; S=B_{c} \omega \\
M_{1}=D\left(\kappa_{1}+\mu \kappa_{2}\right) ; M_{2}=D\left(\kappa_{2}+\mu \kappa_{1}\right) ; H=D_{c} \tau
\end{array}\right.
$$

where N1, N2 are the inner stress-strain forces in the direction of the generatrix and the circumference, respectively; $\mathrm{S}$ is the shear force; M1, M2 are the bending moments acting in 
the planes of the generatrix and the guide, respectively; $\mathrm{H}$ is the torque; $\mathrm{B}, \mathrm{Bc}, \mathrm{D}, \mathrm{Dc}$ are the cylindrical stiffness of the shell in stress-strain, shear, bending and torsion; $\mu$ is the Poisson ratio.

The stresses in the shell are:

$$
\sigma_{11}=\frac{N_{1}}{h} \pm \frac{6 M_{1}}{h^{2}} ; \sigma_{22}=\frac{N_{2}}{h} \pm \frac{6 M_{2}}{h^{2}} ; \sigma_{12}= \pm \frac{6 H}{h^{2}},
$$

where $\sigma 11, \sigma 22$ are the normal stresses in the directions of the generatrix and guide; $\sigma 12$ is the shearing stress; $h$ is the shell thickness.

The action of the contact force FK from the striker, which can be considered either distributed over the area or concentrated, which goes into the safety factor, is taken as an outer influence.

Let us take a displacement approximation similar to that used in [9], which corresponds to the form of dent formation:

$$
w=\frac{1}{4} \sum_{m=1}^{\infty}\left(1-\cos \frac{2 m \pi x}{a}\right)\left(1-\cos \frac{2 m \pi \beta}{b}\right) w_{m},
$$

where, wm - is the amplitude of the member of the displacement series with the number m.

The resolving system of equations is based on the Lagrange principle:

$$
\frac{d \Pi}{d \vec{w}_{m}}-\frac{d A}{d \vec{w}_{m}}=0
$$

where, $\Pi$ - is the potential energy of deformation; A is the work of the outer forces; $\vec{w}_{m}=\left\{w_{1}, w_{2}, \ldots\right\}$ is the vector of amplitudes of a number of displacements.

Due to the nonlinearity of deformations, equation (5) leads to a system of nonlinear equations of the following form:

$$
\left[r^{I}\right] \vec{w}_{m}+\left[r^{I I}\right] \vec{w}_{m}^{I I}+\left[r^{I I I}\right] w_{m}^{I I I}=\vec{p},
$$

where $\left[r^{I}\right],\left[r^{I I}\right],\left[r^{I I I}\right]$ are the coefficient matrices obtained in [9]; $\vec{w}_{m}^{I I}=$ $\left\{w_{1}^{2}, w_{2}^{2}, \ldots\right\} ; \vec{w}_{m}^{I I I}=\left\{w_{1}^{3}, w_{2}^{3}, \ldots\right\} ; \vec{p}$ is the vector of outer loads.

The vector of outer loads is found as a derivative of the work of outer forces with respect to the amplitude vector $\vec{w}_{m}^{I}$. The work of outer forces is equal to:

$$
A=-\int_{0}^{a} \int_{0}^{R b} \frac{F_{\kappa}}{x_{\kappa} R \beta_{\kappa}} \cdot w d x R d \beta .
$$

Substituting in (7) the approximation of displacements (4) and taking the derivative, we obtain an expression for the components of the vector of external loads:

$$
p_{m}=-\frac{F_{\kappa}}{4 x_{\kappa} \beta_{\kappa}}\left(x_{\kappa}-\frac{a}{m \pi} \sin \frac{m \pi x_{\kappa}}{a}\right) \cdot\left(\beta_{\kappa}-\frac{b}{m \pi} \sin \frac{m \pi \beta_{\kappa}}{b}\right)
$$

If the contact force is considered to be applied concentrated, then the work will be equal to $A=-\left.F_{\mathrm{K}} \cdot w\right|_{x=\frac{a}{2} ; \beta=\frac{b}{2}}$, and $p_{m}=-F_{\mathrm{K}}$. It should be noted that such an assumption will lead to overestimated results.

The matrix equation (6) is solved numerically.

The aim of the calculation at this stage is to determine the conditions under which the stresses in the area under study reach the yield point. For the next stage of the calculation, the initial data is a value of the elastic deformation energy Пel. 


\subsection{Modeling the plastic phase of deformation}

Let us consider the plastic phase of deformation development. At this stage, it is assumed that stresses equal to the yield point appear in the dent formation zone. The design scheme of the dent is shown in Fig. 2. The dent is approximated by a truncated tetrahedral pyramid. The sizes of the lower base are a Rb; the sizes of the upper one are xk R $\mathrm{k}$. At the beginning of this phase of deformation, the dent is shown by solid lines, the initial height is equal to w0. The deformed dent is shown with dashed lines. Its height is w0 $+\Delta \mathrm{w}$. The angles at the base are $\sqcup \sqcup \sqcup$ and $\sqcup \sqcup$.

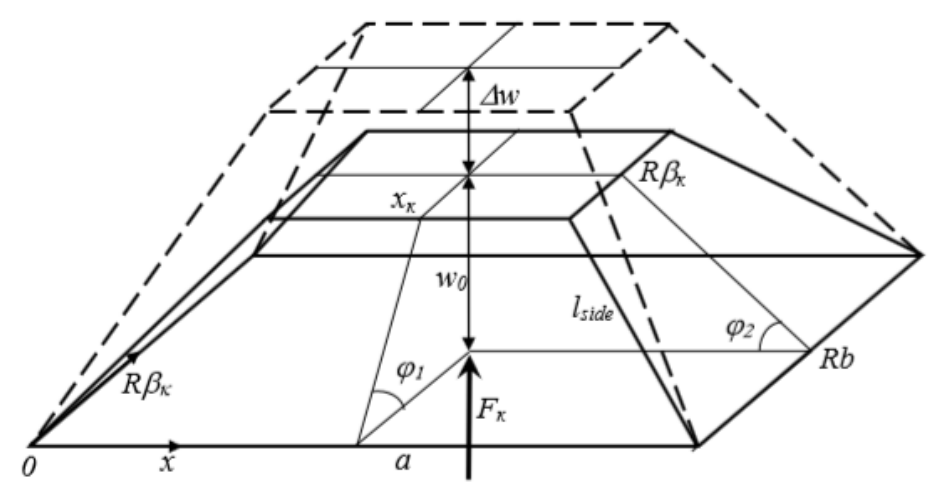

Fig. 2. A scheme of the dent in the plastic phase.

In the process of dent deepening, the deformation energy is spent on stretching the side faces and turning the faces around all edges (plastic hinges). The total energy of the dent formation is:

$$
\begin{aligned}
\Pi_{s h} & =\iint_{S_{\text {side1 }}} P_{m} \varepsilon d S+\int_{0}^{2\left(a+x_{k}\right)} M_{m} \Delta \phi_{1} d x+ \\
& +\int_{0}^{2\left(b+\beta_{\kappa}\right)} M_{m} \Delta \phi_{2} R d \beta+4 M_{m} \Delta \phi_{3} l_{\text {side }}
\end{aligned}
$$

where $\mathrm{PT}_{\mathrm{T}}=\sigma \mathrm{Th}$ is the inner strain force under leak conditions; $M_{\mathrm{T}}=\frac{\sigma_{\mathrm{T}} h^{2}}{4}$ is the bending moment under leak conditions; $\sigma \mathrm{T}$ is the material yield point; $\varepsilon$ is the strain deformation; Sside is the value of the total area of the side faces at the beginning and at the end of this phase of deformation; lside is the length of the side rib; $\varphi 3$ is the angle between the adjacent side faces; $\Delta \varphi 1, \Delta \varphi 2, \Delta \varphi 3$ are the changes in the corresponding angles in the process of deformation.

Consider deformations and angles of rotation in expression (9) on the basis of the pyramid geometry.

$$
\varepsilon=\frac{S_{\text {side } 2}-S_{\text {side } 1}}{S_{\text {side } 1}}
$$

where $\varepsilon=\frac{S_{\text {side } 2}-S_{\text {side1 } 1}}{S_{\text {side } 1}} S_{\text {side } 1}=\left(a+x_{\mathrm{K}}\right) \sqrt{\frac{R^{2}\left(b-\beta_{\mathrm{K}}\right)^{2}}{4}+w_{0}^{2}}+R\left(b+\beta_{\mathrm{K}}\right) \sqrt{\frac{\left(a-x_{\mathrm{K}}\right)^{2}}{4}+w_{0}^{2}}$; and $S_{\text {side } 2}=\left(a+x_{\mathrm{K}}\right) \sqrt{\frac{R^{2}\left(b-\beta_{\mathrm{K}}\right)^{2}}{4}+\left(w_{0}+\Delta w\right)^{2}}+R\left(b+\beta_{\mathrm{K}}\right) \sqrt{\frac{\left(a-x_{\mathrm{K}}\right)^{2}}{4}+\left(w_{0}+\Delta w\right)^{2}}$ are the value of the total area of the side faces at the end of this phase of deformation.

The angles of rotation around the edges can be calculated by the following formulas: 


$$
\begin{gathered}
\Delta \varphi_{1}=\operatorname{arctg} \frac{2\left(w_{0}+\Delta w\right)}{R\left(b-\beta_{\kappa}\right)}-\operatorname{arctg} \frac{2 w_{0}}{R\left(b-\beta_{k}\right)} ; \\
\Delta \varphi_{2}=\operatorname{arctg} \frac{2\left(w_{0}+\Delta w\right)}{a-x_{\kappa}}-\operatorname{arctg} \frac{2 w_{0}}{a-x_{\kappa}} ; \\
\Delta \varphi_{3}=\arccos \left\{\cos \left[\operatorname{arctg} \frac{2\left(w_{0}+\Delta w\right)}{a-x_{\kappa}}\right] \cdot \cos \left[\operatorname{arctg} \frac{2\left(w_{0}+\Delta w\right)}{R\left(b-\beta_{\kappa}\right)}\right]\right\}- \\
-\arccos \left\{\cos \left[\operatorname{arctg} \frac{2 w_{0}}{a-x_{\kappa}}\right] \cdot \cos \left[\operatorname{arctg} \frac{2 w_{0}}{R\left(b-\beta_{k}\right)}\right]\right\} .
\end{gathered}
$$

The lengths of the side ribs are equal to:

$$
l_{\text {side }}=\sqrt{\frac{R^{2}\left(b-\beta_{k}\right)^{2}+\left(a-x_{K}\right)^{2}}{4}+w_{0}^{2}}
$$

Let us determine the work required to destroy the shell by cutting out a rectangular plate along the perimeter of the striker (with the sizes хк by $\mathrm{R} \sqcup \kappa$ ). We assume that along the shell thickness there is a place of the cut stress $\sqcup$ cut. Then the work done by the cutting force of displacement $\Delta \mathrm{w}$ will be equal to

$$
A_{c u t}=2\left(x_{\kappa}+R \beta_{\kappa}\right) h \tau_{c u t} \Delta w
$$

Both the deformation energy (9) and the work (15) are expressed by a change in the pyramid height $\Delta \mathrm{w}$. The resolving equation is compiled with the use of the energy principle:

$$
\frac{d \Pi_{s h}}{d(\Delta w)}-\frac{d A_{c u t}}{d(\Delta w)}=0
$$

After substituting relations (10) - (14) into expression (9) and calculating the derivatives with respect to $\Delta \mathrm{w}$ from equation (16), we obtain a transcendental equation (17).

Equation (17) is solved numerically. As a result, a limiting value of displacement at which destruction occurs is determined. This value is substituted into expression (9) taking into account (10) - (14) and a limiting value of the energy Пsh at the plastic stage of dent formation is found.

$$
\begin{aligned}
& \frac{2 P_{m}\left(a+x_{\kappa}\right)\left(w_{0}+\Delta w\right)}{\sqrt{R^{2}\left(b-\beta_{\kappa}\right)^{2}+4\left(w_{0}+\Delta w\right)^{2}}}+\frac{2 P_{m} R\left(b+\beta_{\kappa}\right)\left(w_{0}+\Delta w\right)}{\sqrt{\left(a-x_{\kappa}\right)^{2}+4\left(w_{0}+\Delta w\right)^{2}}}+ \\
& +\frac{16 M_{m}\left(a+x_{\kappa}\right) R\left(b-\beta_{\kappa}\right)\left(w_{0}+\Delta w\right)}{R^{2}\left(b-\beta_{\kappa}\right)^{2}+4\left(w_{0}+\Delta w\right)^{2}}+\frac{16 M_{m} R\left(b+\beta_{\kappa}\right)\left(a-x_{\kappa}\right)\left(w_{0}+\Delta w\right)}{\left(a-x_{\kappa}\right)^{2}+4\left(w_{0}+\Delta w\right)^{2}}+ \\
& +\frac{8 M_{m} l_{\text {side }}\left(a-x_{\kappa}\right)\left(w_{0}+\Delta w\right)}{\left(a-x_{\kappa}\right)^{2}+4\left(w_{0}+\Delta w\right)^{2}} \\
& \cdot \frac{\sin \left[\operatorname{arctg} \frac{2\left(w_{0}+\Delta w\right)}{a-x_{\kappa}}\right] \cdot \cos \left[\operatorname{arctg} \frac{2\left(w_{0}+\Delta w\right)}{R\left(b-\beta_{\kappa}\right)}\right]}{\sqrt{1-\cos ^{2}\left[\operatorname{arctg} \frac{2\left(w_{0}+\Delta w\right)}{a-x_{\kappa}}\right] \cdot \cos ^{2}\left[\operatorname{arctg} \frac{2\left(w_{0}+\Delta w\right)}{R\left(b-\beta_{\kappa}\right)}\right]}}+ \\
& +\frac{8 M_{m} l_{\text {side }} R\left(b-\beta_{\kappa}\right)\left(w_{0}+\Delta w\right)}{R^{2}\left(b-\beta_{\kappa}\right)^{2}+4\left(w_{0}+\Delta w\right)^{2}} \\
& \cos \left[\operatorname{arctg} \frac{2\left(w_{0}+\Delta w\right)}{a-x_{\kappa}}\right] \cdot \sin \left[\operatorname{arctg} \frac{2\left(w_{0}+\Delta w\right)}{R\left(b-\beta_{\kappa}\right)}\right] \\
& \cdot \frac{\sqrt{1-\cos ^{2}\left[\operatorname{arctg} \frac{2\left(w_{0}+\Delta w\right)}{a-x_{\kappa}}\right] \cdot \cos ^{2}\left[\operatorname{arctg} \frac{2\left(w_{0}+\Delta w\right)}{R\left(b-\beta_{\kappa}\right)}\right]}}{2}= \\
& =2\left(x_{\kappa}+R \beta_{\kappa}\right) h \tau_{\text {cut }} \text {. }
\end{aligned}
$$




\subsection{Final calculations}

At the final stage of calculations, the resulting values of the main parameters corresponding to the limiting state, i.e. boiler shell destruction, are defined.

The limit depth of the dent is a sum of the central deflection values obtained for the elastic and plastic stages of deformation:

$$
w_{\max }=w_{0}+\Delta w .
$$

The limit energy of the dent formation is found by summing the total values of the both calculation stages:

$$
\Pi_{\max }=\Pi_{e l}+\Pi_{s h} .
$$

The limit (permissible) speed of impact is found from the condition that the total energy of the dent formation is equal to the kinetic impact energy:

$$
V_{h}=\sqrt{\frac{2 \Pi_{\max }}{m_{h}}} .
$$

For the limit speed to be calculated, the mass of a really moving body should be taken as the mass: when the tank boiler falls it is the mass of the tank with cargo, when an outer object hits the boiler it is the mass of this object.

\section{Discussion}

The proposed method is intended for modeling the process of emergency collisions of tank boilers and covers the diverse variants of emergency situations. Despite the complexity of the equations being formed this method is very convenient and effective for computational realization and requires insignificant resources in RAM and time. This allows the method to be implemented in any software environment.

Depending on the real conditions of emergency collisions, the different ratios of energy that goes into the elastic and plastic phases of deformation are possible. The situations are possible when the contribution of the initial elastic phase is relatively small and it can be neglected.

On the other hand, there may be conditions under which the main part of energy falls on the elastic phase after which destruction occurs almost immediately.

\section{Conclusions}

A method for mathematical modeling of the behavior of the tank boiler shell during an emergency collision has been developed.

The method makes it possible to estimate the conditions of shell damage with hole formation according to such characteristics as the mass and speed of the striking body, the depth and energy of the dent from the impact.

This method can be applied for design construction of railway tank cars for dangerous goods in order to develop systems of boiler protection from mechanical emergency effects.

Moreover, the method can be used for expertise of real emergencies to find out their causes. 


\section{References}

1. Bespalko S et al 2020 IOP Conf. Ser.: Mater. Sci. Eng. 883012134 doi. org /10.1088/1757-899 X/883/1/01213 4

2. Filin A P 1987 Elements of the theory of shells (L.: Stroyizdat.) p 384

3. Volmir A S 1972 Nonlinear dynamics of plates and shells (Moscow: Nauka) p 432

4. Loytsyansky L G, Lurie A I 1942 Course of Theoretical Mechanics. Part 2 (L. - M.: OGIZ) p 580

5. Bespalko S V 2000 Development and analysis of models of damaging effects on tank barrels for transportation of cryogenic products. Abstract Diss. Dr. Tech. Sciences (Moscow: MIIT)

6. Bespalko S V, Kornienko N A, Chugunov G F 2001 VNIIZhT Bulletin 231

7. Chugunov G F, Bespalko S V 2000 Train traffic safety, Proceedings of the Second Scientific and Practical Conference (M.: MIIT)

8. Volkov V A, Bubnov V M, Bogamaz G I 2000 Transport 625

9. Maslov I G 2016 State of the tank boiler under fire in an emergency: Dis. candidate tech. Sciences (Moscow: Mosk. state un-t ways of communication. Emperor Nicholas II) $\mathrm{p} 132$

10. Grigoriev P S, Ibodulloev Sh R, Poyonov V B 2019 An approach for estimating critical temperatures of buckling of shallow cylindrical shell https://uzjournals.edu.uz/btstu/vol2019/iss2/9

11. Filipov V N, Shebeko Yu N, Ponomarev V M, Plitsina O V 2017 Modeling of the behavior of the railway LPG tank in the hearth flame https://doi.org/10.18322/PVB.2017.26.11.41-51

12. Grigoriev P S 2015 Transp. Russ. Fed. 344

13. Andriyanov S S 2006 The loading of elements of specialized cars equipped with shock absorbers of increased energy intensity: Abstract of Diss Cand. Tech. Sci (Moscow: MIIT)

14. Iman Hazrati Ashtiani, Subhash Rakheja, A K W Ahmed, Jiming Z 2004 Hunting Analysis of a Partially-Railways Tank Car https://doi.org/DOI:10.1115/JRC2015-5631

15. Karpychev V A, Bespalko S V The method of Approximate Esmitation of the Dynamic Load of the Train in the Braking Process https://doi.org/10.1109/FarEastCon.2019.8933873 